\title{
The faux profil (oblique view) of the hip in the standing position. Contribution to the evaluation of osteoarthritis of the adult hip
}

\author{
Michel G Lequesne, Jean-Denis Laredo
}

\begin{abstract}
Objective-The technique and results of a special oblique radiograph of the hip called the "faux profil" (FP) of the hip are described. The FP was evaluated for the detection of joint space narrowing in incipient osteoarthritis of the hip (OAH) as compared with the anteroposterior (AP) radiograph.

Methods-58 hips with incipient osteoarthritis (joint space narrowing, $0-25 \%$ on the AP view) in 48 patients were identified among 200 consecutive patients fulfilling American College of Rheumatology criteria for OAH. Joint space narrowing was measured on the AP and FP radiographs of these 58 hips.
\end{abstract}

Results-The FP view provides a true lateral projection of the femoral head and neck, and an oblique view of the acetabulum tangential to its superoanteromedial edge. On this view, the width of the anterosuperior and posteroinferior parts of the joint space can be measured and compared. Among the $\mathbf{5 8}$ hips with incipient OAH, $36(62 \%)$ showed joint space narrowing on the AP view and 51 (91\%) on the FP view. Among the 22 hips without joint space narrowing on the AP radiograph, $16(72.7 \%)$ showed joint space narrowing on the FP view, involving the anterosuperior part of the joint in 11 cases and the posteroinferior part in five cases.

Conclusion-The FP view in the standing position should be used in incipient OAH as a complement to the AP view in patients with suspected OAH but no joint space narrowing on the AP radiograph. In this situation, nearly three quarters of hips in the study had joint space narrowing on the FP view, usually in the anterosuperior part and less often in the posteroinferior part of the joint. (Ann Rheum Dis 1998;57:676-681)

Department of Radiology, Bone and Joint Imaging Unit, Lariboisière Hospital (Assistance Publique des Hôpitaux de

Paris), Paris, France J-D Laredo

Correspondence to: Professor M G Lequesne, 31-33 rue Guilleminot, 75014, Paris, France.

Accepted for publication 24 July 1998 taken in the supine as compared with the softening caused by degenerative joint disease results in a small but significant increase in joint space width in the absence of joint loading.

The "faux profil" (FP) view of the hip in the standing position was first proposed in 1961 for the diagnosis of certain forms of osteoarthritis of the hip (OAH) and of developmental dysplasia of the hip. ${ }^{5}$ It was quickly accepted by radiologists, rheumatologists, and orthopaedic surgeons in France and in some European ${ }^{6-9}$ and Canadian ${ }^{10}$ centres as a useful tool for the early diagnosis of $\mathrm{OAH}$ and for the preoperative evaluation of congenital dysplasia and subluxation of the hip. Recently, American radiologists have also mentioned the FP among radiographic views useful in the evaluation of the adult dysplastic hip. ${ }^{11}$

Although both OAH and developmental dysplasia of the hip are indications for the FP view, only the former is discussed here. JSN, an important feature of $\mathrm{OAH}$, is sometimes absent on the anteroposterior (AP) radiograph. We conducted this study to determine the proportion of cases of incipient $\mathrm{OAH}$ in which unequivocal JSN is seen only on the FP view.

\section{Methods}

TECHNIQUE

The radiographic table is vertical. The cassette is inserted in the table rack. Ideally, fluoroscopy is used to ensure that the projection is correct, although experienced technicians can obtain correct FP views without fluoroscopy.

The patient first stands sidewise with the hip to be studied in contact with the radiographic table. The axis of the ipsilateral foot (second metatarsal bone) is parallel to the radiographic table and must remain in this position during the subsequent phase to obtain a true lateral view of the proximal end of the femur (fig 1A). The pelvis is then rotated $25^{\circ}$ backwards (fig $1 \mathrm{~B}, \mathrm{C})$ to profile the anterosuperomedial edge of the acetabulum. The back of the patient is at a $65^{\circ}$ angle with the radiographic table. A wedge may be placed between the patient's back at the level of the superior border of the sacrum and the radiographic table (fig $1 \mathrm{~B}, \mathrm{C}$ ) to check that positioning is correct.

The $x$ ray beam is centred on the medial part of the contralateral groin. If fluoroscopy control is used, the $x$ ray beam is centred between the two femoral heads. The focus-film distance is $110 \mathrm{~cm}$.

Correct positioning is checked using the following criteria (fig 2A, B): the anterosuperior part of the pubis projects near the anterior edge of the greater trochanter; the distance between 
A

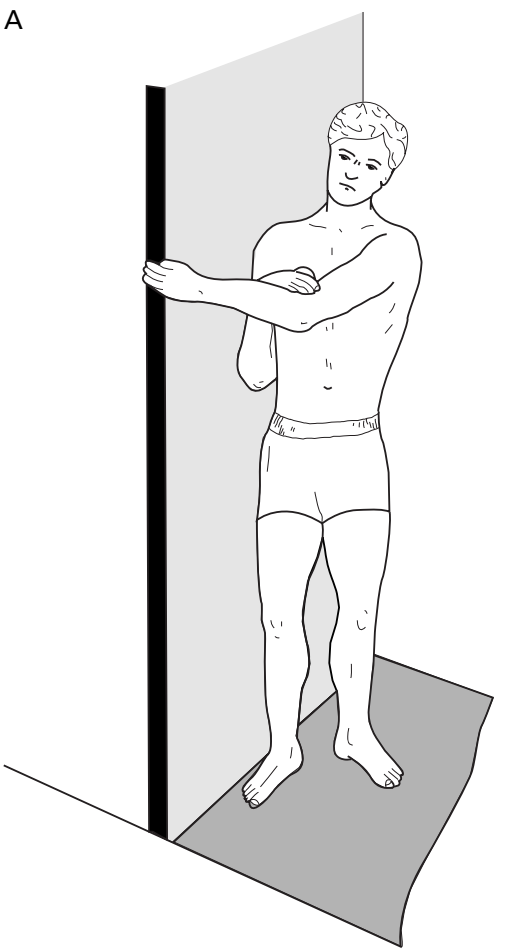

B

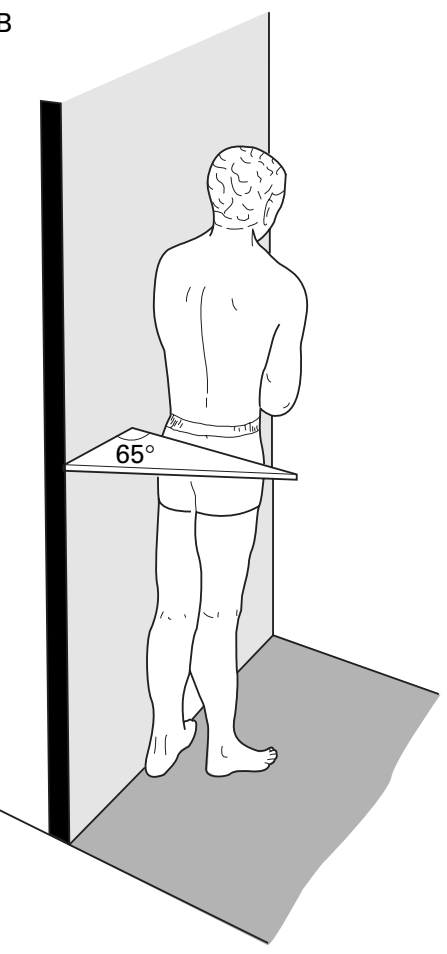

C

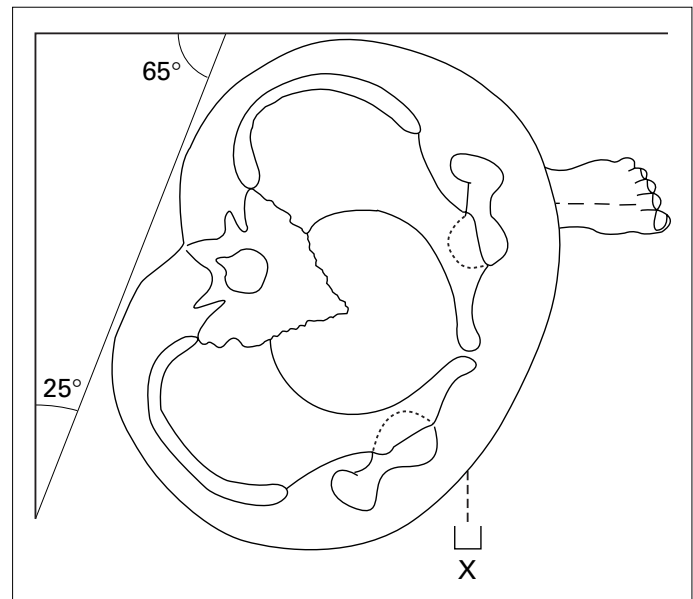

Figure 1 Diagram of the patient's position. (A) Position of the patient for an FP radiograph of the right hip. Axis of the foot (2nd metatarsus) should be parallel to the radiographic table. (B) For the left hip. (C) Cross sectional diagram of the pelvis showing: (a) the $25^{\circ}$ angle between the anterior edge of the acetabulum and the coronal plane; (b) the position of the pelvis, rotated $25^{\circ}$ backward around a vertical axis to align the

superoanteromedial edge of the targeted acetabulum with the $x$ ray beam; (c) the position of the lower limb with the axis of the foot parallel to the film so as to obtain a true lateral view of the proximal third of the femur.

the two femoral heads is between two and three thirds of the diameter of the targeted femoral head; the centre of the femoral head, the axis of the femoral neck, and the axis of the femoral shaft are approximately on the same vertical line; the lesser trochanter is slightly prominent posteriorly, which indicates neutral rotation of the lower limb; the posterior margin of the greater trochanter projects just behind the posterior edge of the femoral neck.

Conversely, the following criteria are indicative of inaccurate positioning: a between heads distance of more than three thirds of the diameter of the ipsilateral femoral head indicates a

position that is too close to a true $45^{\circ}$ oblique view; a between heads distance of less than two thirds of the diameter of the ipsilateral femoral head indicates a position too close to a true lateral view; exaggerated prominence of the lesser trochanter indicates internal rotation of the lower limb; absent or minimal prominence of the lesser trochanter indicates external rotation of the leg.

COMPARISON OF JOINT SPACE WIDTH ON THE ANTEROPOSTERIOR AND FAUX PROFIL VIEWS IN $\mathrm{OAH}$

The AP radiographs of 200 consecutive patients (118 female and 82 male with a mean age of 62.2 years; range 37.5-78) meeting classification tree 2 of the American College of Rheumatology (ACR) criteria for painful $\mathrm{OAH}$ - that is, chronic pain and at least osteophytosis $^{12}$ - were reviewed to select those with incipient OAH. The latter was defined as presence on the AP view of osteophytosis and mild ( $\leqslant 25 \%)$, doubtful, or absent JSN. An FP view of every incipient OA hip was obtained. Joint space measurement (chondrometry) was performed using calipers and a ruler graduated in half millimetres, as described previously. ${ }^{13}$ JSN was evaluated by comparison with the healthy side. Cases with bilateral OAH were included when they fulfilled one of the three following criteria: decreased joint space as compared with an earlier radiograph, if available; joint space width on the AP view of $3 \mathrm{~mm}$ or less, a threshold used in previous studies ${ }^{14}{ }^{15}$; mild JSN compared with the contralateral hip when the latter exhibited osteophytosis without JSN. In cases with probable JSN difficult to assess because of the lack of a possible comparison, the JSN was classified as "doubtful".

One patient in this series had a FP view taken during arthrography. This was compared with a normal arthrographic FP view obtained in an asymptomatic patient as part of a previous study. ${ }^{16}$

\section{Results}

\section{NORMAL HIP}

The FP view provides a true lateral view of the proximal end of the femur, and also profiles the anterosuperomedial edge of the acetabulum as a cortical line crossing the femoral head projection almost vertically. Figure 2 shows the main landmarks of the normal hip in FP view.

In the normal hip, JS width is greater at the anterosuperior than at the posterior part of the joint (figs 2 and 6).

The axis of the femoral neck is nearly vertical in the absence of significant anteversion or retroversion (fig 2B).

JOINT SPACE NARROWING

Forty eight of the 200 patients were found to have incipient $\mathrm{OAH}$, which was bilateral in 10 patients.

Among the 58 cases of incipient $\mathrm{OAH}$, $36(62 \%)$ exhibited JSN on the AP view and $51(91 \%)$ on the FP view. JSN was apparent on the FP view in 35 of the 36 hips with a JSN on the AP radiograph. In $22(38 \%)$ hips, 
JSN was doubtful or absent on the AP view (table $1(\mathrm{a})$ ). In $16(72.7 \%)$ of these 22 hips, the FP view showed definite JSN in either an anterosuperior $(n=11)$ or a posterior or posteroinferior $(n=5)$ location (table $1(b))$. In

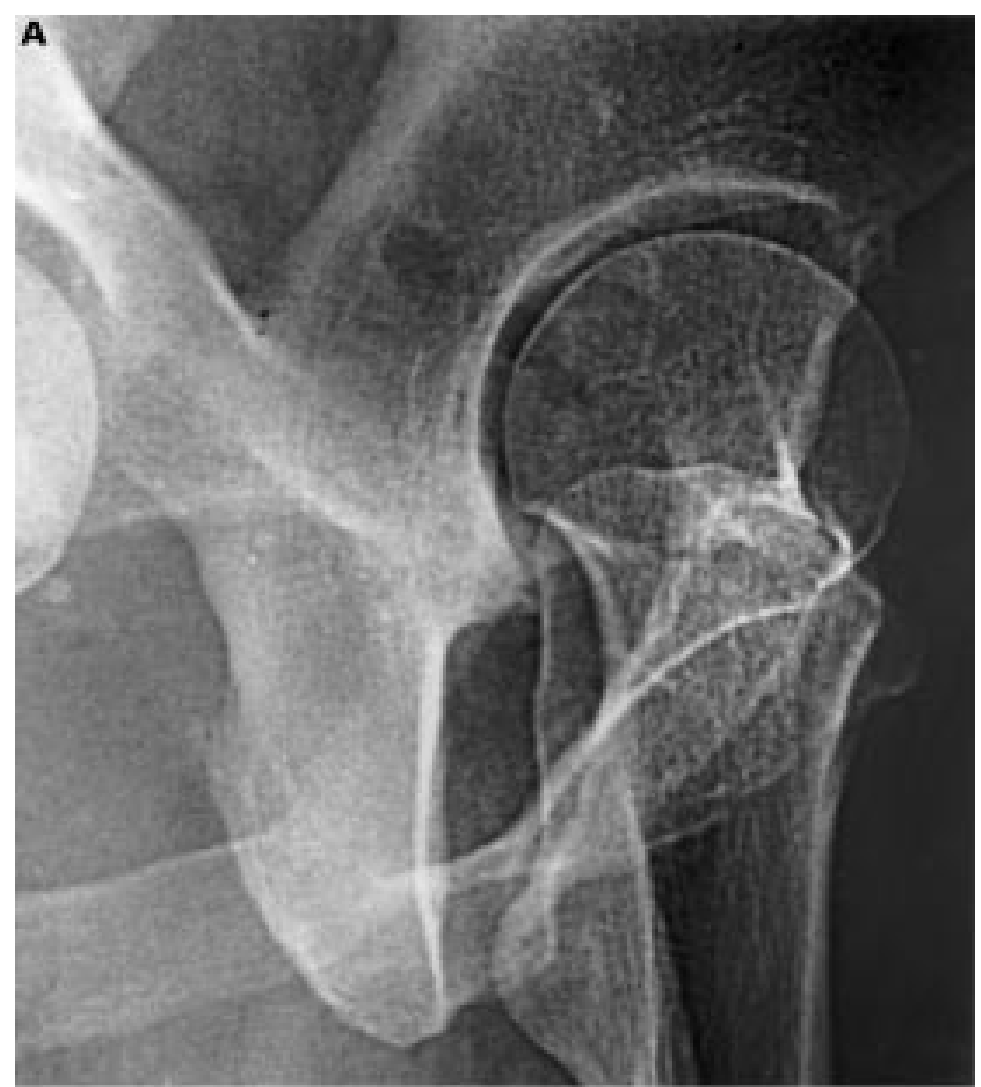

日

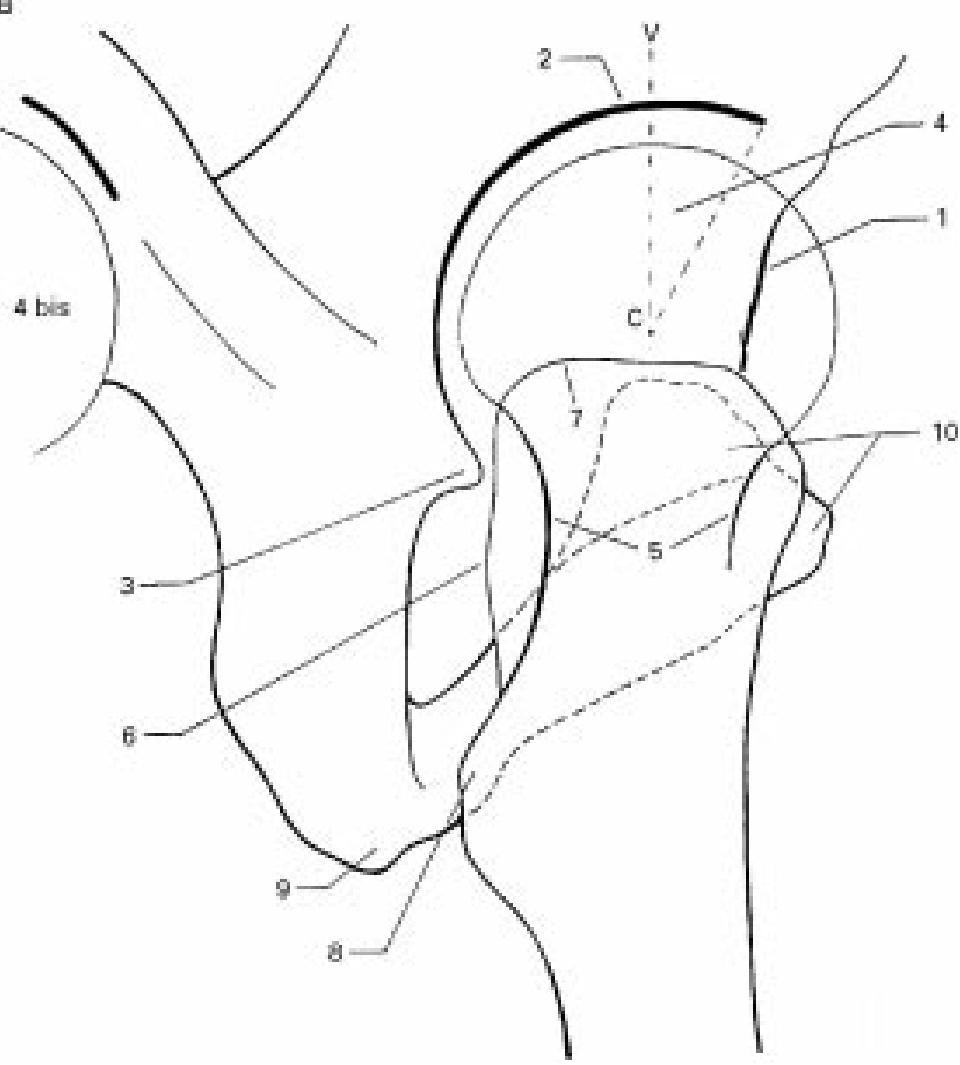

Table 1 FSN on (a) the AP view, (b) the FP view

\begin{tabular}{lcc}
\hline & Number & $\%$ \\
\hline (a) JSN on the AP view in 58 patients & with incipient & OAH \\
superolateral JSN & 21 & 36.2 \\
superior & 7 & 12.0 \\
superomedial and medial & 8 & 13.8 \\
doubtful & 10 & 17.2 \\
absent & 12 & 20.6 \\
(b) JSN on the FP view in the 22 cases of OAH with doubtful \\
or absent JSN on the AP view & & \\
anterosuperior JSN & 11 & 50.0 \\
posterior & 5 & 22.7 \\
doubtful (3 post, 1 ant-sup) & 4 & 18.0 \\
absent & 2 & 9.2 \\
\hline
\end{tabular}

four $(18 \%)$ cases, the FP view showed doubtful JSN (anterosuperior, $\mathrm{n}=1$; posteroinferior, $\mathrm{n}=3$ ).

Anterosuperior $\mathcal{F S N}$

Figures 3, 4, 5 show examples of anterosuperior JSN visible only on the FP view. Special care was taken to identification of a widening of the posteroinferior part of the joint space (fig $3 \mathrm{~B}, 4 \mathrm{~B}$ ), an abnormality that indicates anterosuperior subluxation of the femoral head. As mentioned above, the JS on the FP view of the normal hip is widest at the anterosuperior part of the joint. It follows that an equal JS width between the anterosuperior and posteroinferior parts of the joint indicates incipient anterior subluxation of the femoral head caused by anterosuperior JSN (fig 4B). In hips with posteroinferior JS widening, JS measurement (chondrometry) consistently demonstrated anterosuperior JSN compared with the contralateral hip. In addition, the FP view helped to localise subchondral bone cysts associated with $\mathrm{OAH}$ (fig 4B).

Arthrography performed in one of our patient exhibits the thinning of the anterosuperior part of the acetabular cartilage, visible only on the FP view (fig 5A, B). A FP view taken during arthrography of a normal, asymptomatic hip shows clearly that the thickness of the acetabular hyaline cartilage increases gradually from the posteroinferior to the anterosuperior part of the joint (fig 6).

Posteroinferior $\mathcal{F S N}$ (fig $7 A, B$ )

All of the five hips with posteroinferior JSN also exhibited osteophytosis of the posterior acetabular horn, which was clearly visible on the $\mathrm{FP}$ view (fig 7B).

Figure 2 Normal findings on the FP view. Radiograph and diagram. (1) Anteromedial edge of the acetabulum; (2) The superior part of the acetabulum appears as a sclerotic curved line ending at point $A$, which is the anterior extremity of the acetabular roof. The posterior part of the acetabulum (that is, the posterior extension of the above mentioned superior sclerotic line) ends inferiorly at the posteroinferior rim - that is, at the acetabular horn (3). The distance between the two femoral heads (4 and 4 bis) is two to three thirds of the diameter of the ipsilateral femoral head. The femoral neck (5) is visible through the greater trochanter. The posterior (6) and the superior edges (7) of the greater trochanter are well delineated. The lesser trochanter (8) appears as a small prominence posterior to the inferior part of the greater trochanter. The ischiopubic ramus joining the ischial tuberosity (9) to the pubic bone (10) is partly superimposed on the proximal femur. The anterosuperior part of the pubic bone (10) is superimposed on the anterior margin of the greater trochanter. 

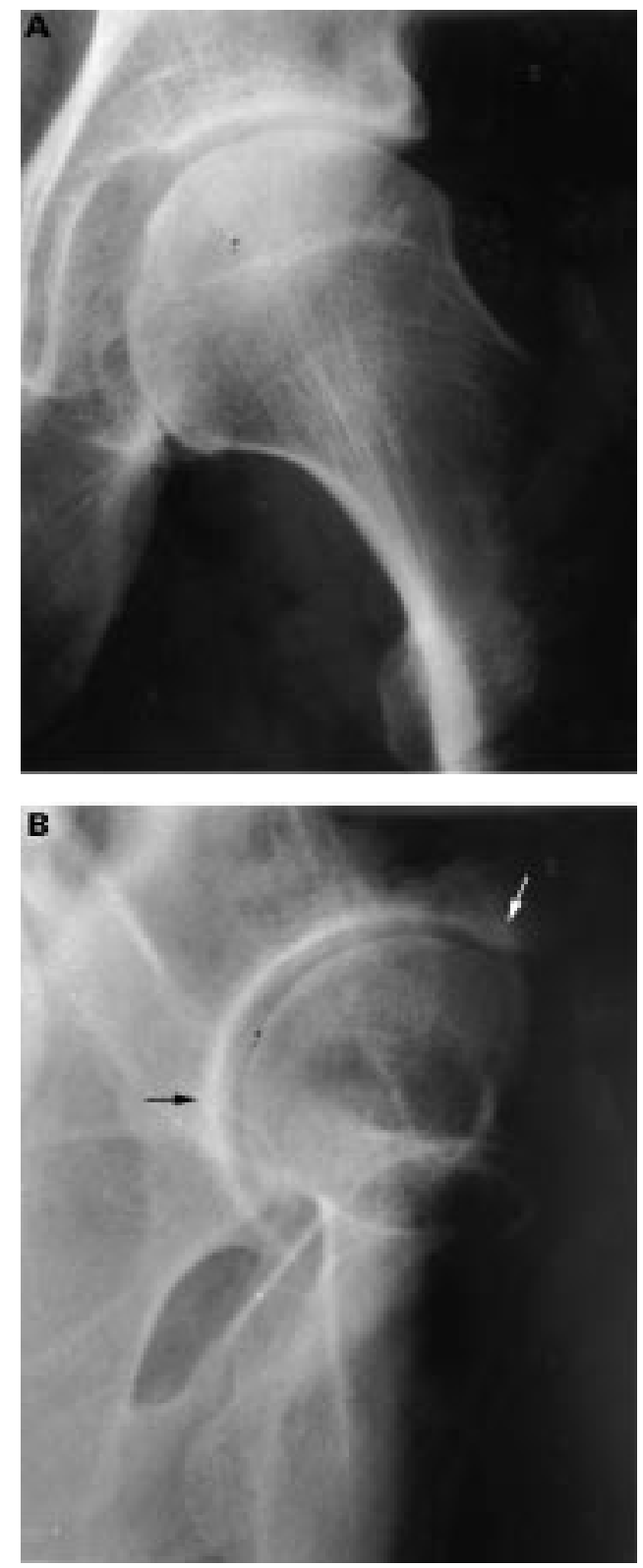

Figure 3 Anteroposterior $(A)$ and faux profil $(B)$ views in incipient $O A H$. (A) In this 50 year old man with clinical $O A H$ characterised by pain, minimal osteophytosis, and a supra-acetabular cyst, the anteroposterior view shows no $\mathcal{H S N}$. $\mathcal{H S N}$ is visible only on the FP view (B), at the anterosuperior part of the joint (white arrow). The posterior FS is widened (black arrow). The femoral head has begun to migrate anteriorly and superiorly.

\section{Discussion}

Among the 22 hips with no JSN on the AP view, the FP view demonstrated a JSN in 16 $(72 \%)$. One explanation to the greater sensitivity of the FP view in detecting JSN is that the FP view permits the comparison of JS width between the superior and the inferior part of the joint, as in the normal hip, JS width is greater in the superior than in the posterior part of the joint (fig 2A, fig 6).

Although in patients with hip pain, osteophytosis alone is sufficient to make a diagnosis of $\mathrm{OAH}$ according to ACR criteria, ${ }^{12}$ presence of JSN establishes the diagnosis with greater confidence. In addition, the FP view may disclose a JSN in cases that do not fulfill ACR criteria
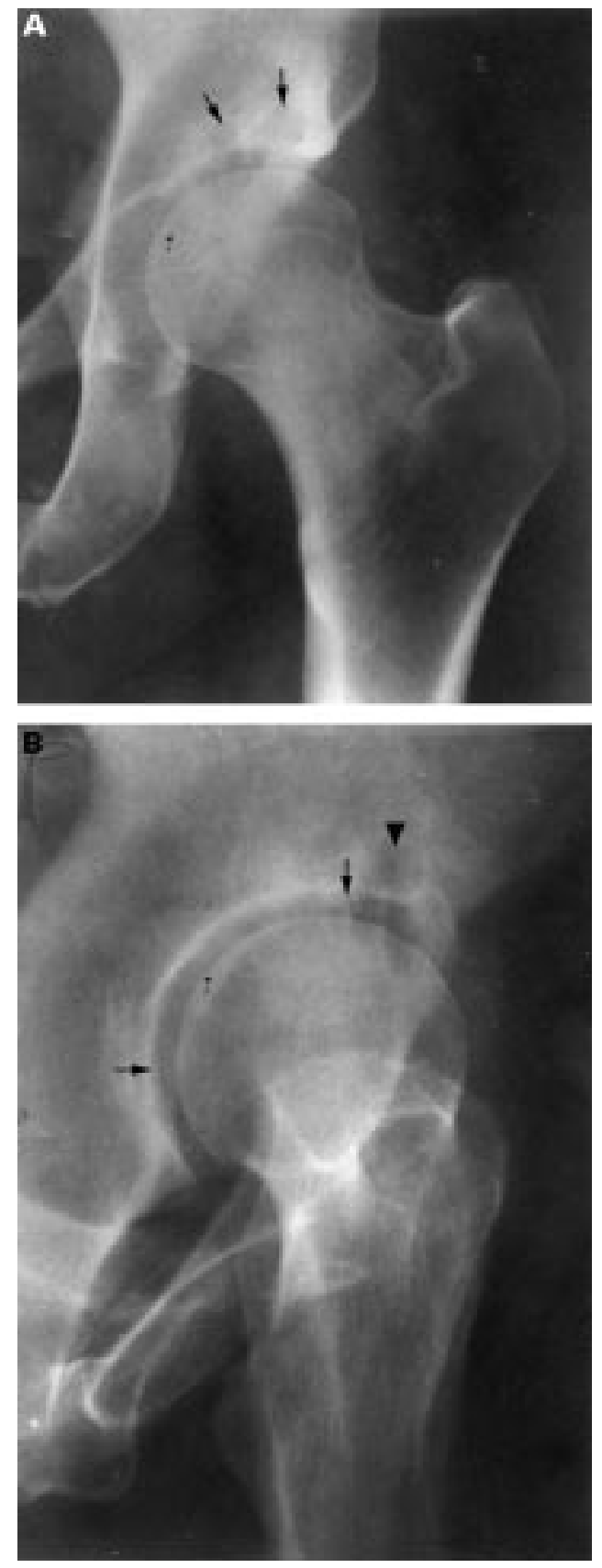

Figure 4 Anteroposterior and FP views in incipient $O A H$. This 54 year old woman with painful left hip subsequently underwent total hip replacement. The pathologist confirmed the diagnosis of typical $O A$ of the hip. (A) On the AP view, neither osteophytosis nor $7 S N$ are visible. The only abnormalities were two cysts in the acetabular roof (short arrows). This case was therefore not included in the series reported in this article because it did not meet $A C R$ criteria. ${ }^{12}$ (B) On the FP view, the equal width of the anterosuperior and posterior portions of the $\mathcal{H S}$ long arrows) already indicates anterosuperior $\mathcal{H S N}$ with incipient anterior subluxation of the femoral head, as the cartilage is normally thicker at the superior than at the posterior part of the joint (fig $2 B$ and fig 6). Subchondral cysts are visible in the same location (short arrow).

for OA of the hip - that is, without JSN, or even osteophytosis, on the AP view. Figure 4A, B provides an example of this situation.

Regarding JSN criteria on the FP view, three points deserve special mention. (1) Comparison with the contralateral hip, if healthy, is very useful in incipient OAH. (2) Differentiating normal JS width from JSN on the FP view is 

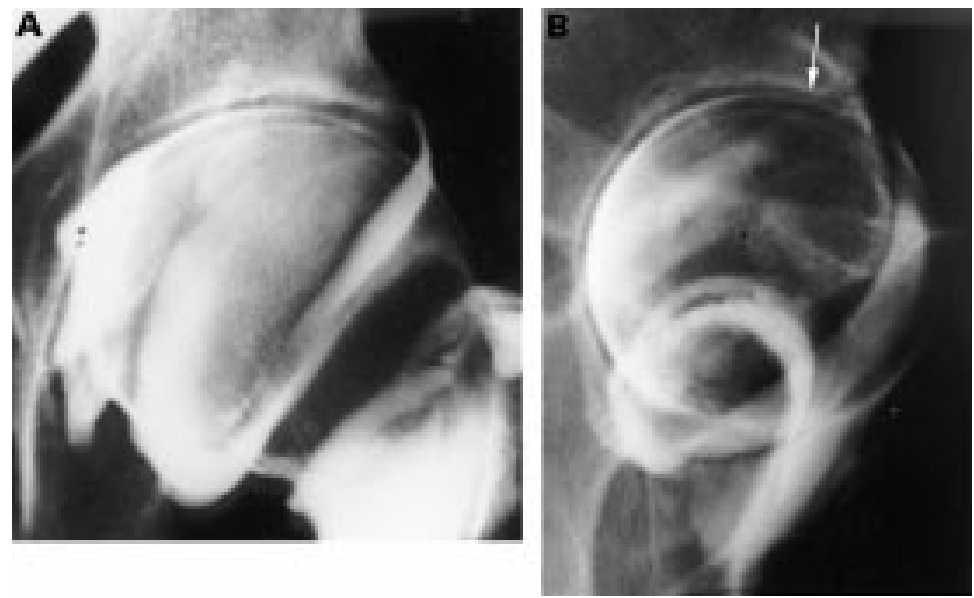

Figure 5 Anteroposterior and faux profile views during arthrography. Incipient $O A$ of the hip. This 58 year old woman had a one year history of pain in her left hip. (A) The AP view does not show cartilage thinning. (B) On the FP view, the width of the acetabular cartilage is markedly reduced at the anterosuperior part of the joint (arrow). The widened posterior FS is filled with contrast medium.

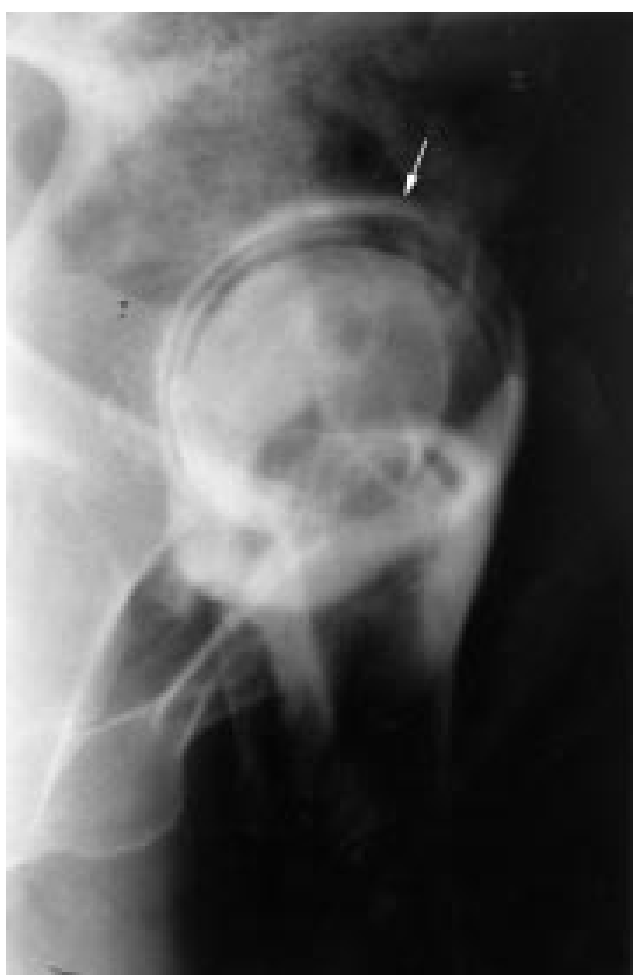

Figure 6 FP view during arthrography. Normal hip. The anterosuperior part of the $\mathcal{F S}$ (especially the acetabular cartilage) is normally thicker (arrow) part. Note the mild acetabular deficiency: the anterior part of the acetabular roof is too short.

more difficult at the posteroinferior than at the anterosuperior part of the joint. Three of the four cases with doubtful JSN on the FP view in our study involved the posteroinferior part of the joint. In difficult cases, presence of an osteophyte arising from the posterior horn is an additional sign of OAH. (3) Reproducibility of the FP view is very good when fluoroscopy is used and has been found to be similar on the FP view as on the AP view. ${ }^{17}$ Reproducibility of radiographic views is of critical importance in randomised controlled trials involving JS width measurement.
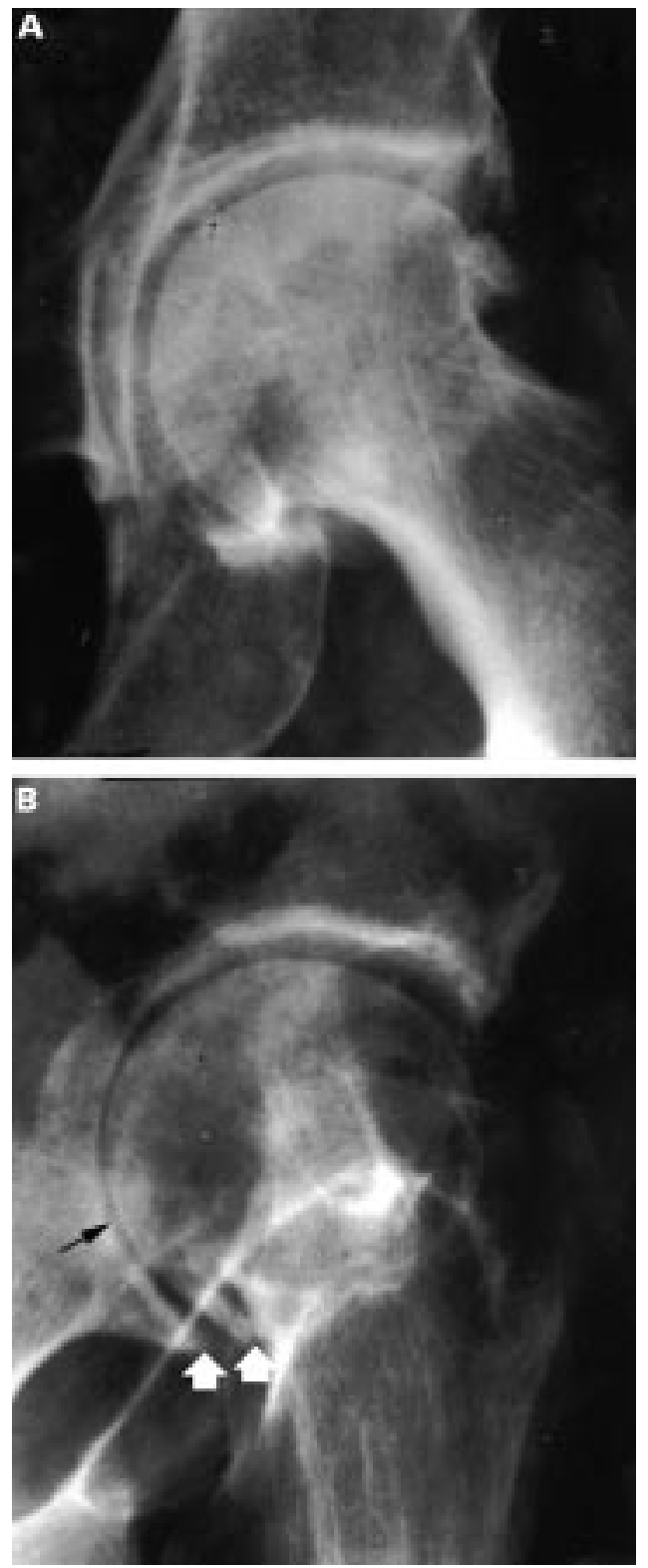

Figure 7 (A) AP view showing only osteophytosis; (B) FP view: posteroinferior $7 S N$ (black arrow). Note also the subchondral sclerosis in the area of $\mathcal{H S N}$, and the osteophytes arising from the posterior horn of the acetabulum and from the posteroinferior part of the femoral head-neck junction (white arrows).

USEFULNESS OF THE FAUX PROFIL VIEW IN OAH

The main circumstances in which we found the FP view to be useful for detecting JSN are: (1) Incipient OAH with no or doubtful JSN on the $\mathrm{AP}$ view (figs $3,4,5$ ) as detailed above. (2) Bilateral OAH precluding comparison of JS width with a healthy side on the AP view. Minimal concentric (without focal narrowing) JSN on the AP view is difficult to identify with confidence in bilateral OAH. In such cases, the FP view can show definite anterosuperior JSN, establishing the diagnosis of OAH. (3) Chondrometry in controlled trials. Combined use of the AP and FP views has recently been shown to increase the sensitivity of JSN detection compared with use of the AP view alone. This is of critical importance in trials evaluating possible "chondroprotective" or structure 
modifying drugs. ${ }^{18}$ In a study of 50 patients with $\mathrm{OAH},{ }^{19}$ JSN was significantly more marked on the FP view (mean (SD) JS width, $2.5(0.9) \mathrm{mm})$ than on the AP view (3.1 (0.10) $\mathrm{mm})(\mathrm{p}=0.001)$. Mean JSN, graded using a four point scale (0-3), was identical on FP and $\mathrm{AP}$ views in $56 \%$ of cases, more marked on the FP view in $32 \%$, and more marked on the AP view in $12 \%$. JSN assessment using the four point scale was more sensitive when both FP and AP views were used. ${ }^{19}$ Although the value of the FP view in controlled trials of structure modifying drugs remains to be precisely determined, the four point JSN grading system applied to the FP and the AP views can easily be used in everyday practice and may improve our ability to set up the prognosis of OA of the hip. In a recent prospective study on 508 patients with $\mathrm{OAH}$, it was shown that the faster the JSN over the year, the sooner the need of hip replacement. ${ }^{20}$

In conclusion, an FP view should be obtained when $\mathrm{OAH}$ is suspected based on presence of osteophytosis and/or subchondral cyst(s) and/or subchondral sclerosis with absent or doubtful JSN on the AP view. JSN may be apparent only on the FP view at the anterosuperior or more rarely the posteroinferior part of the joint. In doubtful cases, comparison with the contralateral hip is useful. We suggest that both the AP and FP views should be used to evaluate the presence of JSN in OAH.

We are grateful to Mrs Wolfe, $\mathrm{MD}$, for reviewing the English of the manuscript.

1 Resnick D. Diagnosis of bone and joint disorders. Philadelphia: W B Saunders, 1988

2 Magilligan DJ. Calculation of the angle of anteversion by means of horizontal lateral roentgenography. J Bone Joint Surg 1956;38A:1231-46.

3 Dunlap K, Shands AR, Hollster LC, Gaul JS, Streit HA. A Bone Joint Surg 1953;38A:289-312.
4 Conrozier T, Lequesne M, Tron AM, Mathieu P, Berdah L, Vignon E. Effect of standing position on the radiographic hip joint space in osteoarthritic patients. Osteoarthritis hip joint space in oste

5 Lequesne M, Sèze S de. Le faux profil du bassin. Nouvelle incidence radiographique pour l'étude de la hanche. Son utilité dans les dysplasies et les différentes coxopathies. Revue du Rhumatisme et des Maladies ostéo-articulaires 1961;28:643-52.

6 Ganz R, Klaue K, Vinh TS, Mast JW. A new periacetabular osteotomy for the treatment of hip dysplasias. Clin Orthop 1988;232:26-36.

7 Trousdale RT, Ekkernkamp A, Ganz R, Wallrichs SL. Periacetabular and intertrochanteric osteotomy for the treatment of osteoarthritis in dysplastic hips. J Bone Joint Surg 1995;77A:73-85.

8 Lemoine A, Briard JL, Lassale B. Le plan de profil de la hanche dans les ostéotomies intertrochantériennes de varihation type Pauwels. Rev Chir Orthop1978;64:558-62.

9 Postel M. Cotyles obliques et butées associées. Rev Chir Postel M. Cotyles obliqu
Orthop 1978;569-72.

10 Zukor D, Lander P. Hip diseases. Young adults. Appropriate imaging studies and their interpretation. Can J Surg 1995;38 (suppl 1):S6-12.

11 Delaunay S, Dussault RG, Kaplan PA, Alford BA. Radiographic measurements of dysplastic adults hips. Skeletal Radiol 1997;26:75-81.

12 Altman R, Alarcon G, Appelrouth D, Bloch D, Borenstein $\mathrm{D}$, Brandt $\mathrm{K}$, et al. The American College of Rheumatology criteria for the classification and reporting of osteoarthritis of the hip. Arthritis Rheum 1991;34:505-14.

13 Lequesne M. Quantitative measurements of joint space during progression of osteoarthritis: "chondrometry". In: Kuettner K, Goldberg V, eds. Osteoarthritic disorders. Ruettner K, Goldberg V, eds. Osteoarthritic disorders. 1995:427-44.

14 Konradsen L, Berg Hansen E M, Sondergaard L. Long distance running and osteoarthrosis. Am J Sports Med 1990; 18:379-81.

15 Lindberg H, Roos H, Gardnell P. Prevalence of coxarthrosis in former soccer players. 286 players compared with matched controls. Acta Orthop Scand 1993;64:165-7.

16 Lequesne M, Becker J, Bard M, Witvoët J, Postel M. Capsuar constriction of the hip: arthrographic and clinical considerations. Skeletal Radiol 1981;6:1-10

17 Conrozier T, Mathieu P, Piperno M, Bochu M, Vignon E. Hip joint space measurement from Lequesne's lateral view is as reproducible as from frontal radiograph. [Abstract]. Rev Rhum Engl Ed 1996;63:747.

18 Altman RD, Brandt K, Hochberg M, Moskowitz R and the OARS committee members. Design and conduct of clinical trials in patients with osteoarthritis. Osteoarthritis Cartilage $1996 ; 4: 217-43$.

19 Conrozier T, Mathieu P, Shan C, Piperno M, Vignon E. Hip lateral radiograph improves the sensitivity of joint space narrowing assessment in hip osteoarthritis (OA). [Abstract]. Rev Rhum Engl Ed 1996;63:747.

20 Dougados M, Gueguen A, Nguyen M, Berdah L, Lequesne $M$, Mazières $\mathrm{B}$, et al. Is decision of total hip replacement (THR) in hip osteoarthritis (OA) an outcome assessment of osteoarthritis? Arthritis Rheum 1997;40 (suppl 9):S280. 